\title{
Physician Counseling for Colorectal Cancer Screening: Impact on Patient Attitudes, Beliefs, and Behavior
}

\author{
Joshua J. Fenton, MD, MPH, Anthony F. Jerant, MD, \\ Marlene M. von Friederichs-Fitzwater, PhD, MPH, Daniel J. Tancredi, PhD, \\ and Peter Franks, MD
}

Purpose: To determine how often primary care physicians address patient-level health behavioral constructs that are associated with colorectal cancer (CRC) screening and whether physician counseling addressing constructs is associated with favorable changes in patients' attitudes, beliefs, intentions, and subsequent screening.

Methods: We conducted a prospective cohort study of patients eligible for CRC screening and attending routine appointments within two academic primary care clinics (50 patients, 20 primary care clinicians). Patients completed validated measures of behavioral constructs associated with CRC screening (benefits, barriers, susceptibility, self-efficacy, intention, and stage of readiness) before and after their visits. Audio-recorded discussions of CRC screening were coded for conversation addressing constructs. Bivariate and regression analyses estimated associations between discussions that did and did not address constructs and, after the visit, measures of perceived benefits, barriers, susceptibility, self-efficacy, intention, and completion of CRC screening within 6 months.

Results: Physicians discussed CRC screening during 38 encounters (76\%) and addressed behavioral constructs during 26 (52\%). Relative to visits without CRC screening discussion, visits with discussion were associated with increased perceived susceptibility $(\beta=0.39 ; 95 \% \mathrm{CI}, 0.09-0.68)$ and screening intention $(\beta=0.42 ; 95 \% \mathrm{CI}, 0.11-0.73)$ after the visit but no significant change in perceived benefits, barriers, or self-efficacy. Within 6 months, 17 of 38 patients (45\%) who discussed screening completed screening compared with 0 of 12 patients who did not discuss screening $(P=.001)$. Associations between discussions and outcomes were similar whether or not counseling addressed behavioral constructs.

Conclusions: These findings suggest that physician counseling is associated with increased patient perception of CRC susceptibility, greater screening intention, and completion of screening regardless of whether counseling addresses behavioral constructs. (J Am Board Fam Med 2011;24:673-681.)

Keywords: Behavioral Counseling, Cancer Research, Colorectal Cancer Screening, Communication

Since the mid-1990s, major public health organizations have unanimously endorsed colorectal cancer (CRC) screening for adults aged 50 to 75

This article was externally peer reviewed.

Submitted 2 January 2011; revised 28 April 2011; accepted 16 May 2011.

From the Departments of Family and Community Medicine (JJF, AFJ, PF), Internal Medicine (MMvF-F), and Pediatrics (DJT) and the Center for Healthcare Policy and Research (JJF, AFJ, DJT, PF), University of California at Davis, Sacramento, CA.

Funding: Supported by a grant from the University of California Cancer Coordinating Committee (JJF) and by National Cancer Institute grant no. 1R01CA131386 (AFJ). years, ${ }^{1-3}$ yet at least $40 \%$ of eligible Americans are not up to date with screening. ${ }^{4}$ A physician's recommendation has been consistently associated with CRC screening, ${ }^{5-7}$ yet little is known about how

Prior presentation: "Characterizing Effective Counseling for Colorectal Cancer Screening," North American Primary Care Research Group Annual Meeting, Seattle, WA, November 14, 2010.

Conflict of interest: none declared.

Corresponding author: Joshua Fenton, Department of Family and Community Medicine, UC Davis Medical Center, 4860 Y Street, Suite 2300, Sacramento, CA 95817 (E-mail: Joshua.fenton@ucdmc.ucdavis.edu). 
physicians recommend screening and how patients respond to different types of recommendations.

Theory-based research has identified patientlevel behavioral or psychological constructs that are associated CRC screening, including perceived benefits of screening, perceived barriers, perceived susceptibility to CRC, and self-efficacy or confidence in carrying out CRC screening. ${ }^{5,8-13}$ Theorybased interventions to modify these constructs also have successfully motivated primary care patient adherence with CRC screening, but these interventions occurred immediately before primary care visits and did not aim to modify physician recommendations. ${ }^{14,15}$ It is plausible that physician recommendations that address known behavioral constructs would be more effective at promoting CRC screening than recommendations that do not.

Within two academic primary care clinics, we audiotaped provider-patient encounters to assess the extent to which providers addressed behavioral constructs related to CRC screening. We hypothesized that (1) conversations related to CRC screening would be associated with advancement in a patient's intention to undergo CRC screening and the ultimate completion of screening; and (2) conversations addressing behavioral constructs relevant to CRC screening would be more strongly associated with CRC screening intention and completion than conversations not addressing constructs.

\section{Methods}

\section{Design, Setting, and Participants}

We performed a prospective cohort study of patients attending appointments at two academic primary care clinics at the University of California, Davis, Medical Center in Sacramento, CA. The clinics are affiliated with family practice and internal medicine residencies, respectively, and providers include faculty, residents, and faculty of a family nurse practitioner/physician assistant training program. We recruited providers during staff meetings and by E-mail and obtained written informed consent to recruit potentially eligible patients. The University of California, Davis, Institutional Review Board approved the study.

From September 2008 to December 2009, research staff reviewed clinic schedules of providers to identify potentially eligible subjects based on age between 50 and 75 years and chart evidence of being eligible for CRC screening (neither fecal occult blood testing [FOBT] during the past year, flexible sigmoidoscopy during the past 5 years, nor colonoscopy in the past 10 years). Staff approached potentially eligible patients by telephone and instructed interested patients to arrive 45 minutes earlier than their appointment times for enrollment. Patients were considered ineligible if they could not speak English or responded that they were attending appointments for acute illness. All patients provided written informed consent and were compensated with a \$20 gift card.

\section{Conceptual Framework}

We used the Transtheoretical Model (TTM) to conceptualize the role of physician counseling in motivating patients' screening decisions. ${ }^{16}$ Within the TTM, patient health behavior is determined by the perceived balance of benefits and barriers of engaging versus not engaging in the behavior, perceived susceptibility to the health condition, and self-efficacy (confidence) in being able to successfully engage in the behavior. The model further conceives that patient intention may proceed dynamically through stages of readiness, from precontemplative (not considering the behavior) to planning (after the patient has contemplated and is ready to engage in the behavior). The TTM and related behavioral models have predictive validity for CRC screening and other cancer screening outcomes. ${ }^{9,11,12,17}$

\section{Measures}

Before and after the visit, patients completed questionnaires regarding the following TTM constructs relevant to CRC screening: perceived benefits and barriers to screening, perceived risk and susceptibility to CRC, self-efficacy for undergoing screening, and intention to complete screening. We constructed before- and after-visit scales for each behavioral construct by averaging across standardized responses to questions from validated multiitem scales. ${ }^{9,10}$ Internal consistencies (Cronbach's $\alpha$ ) for the scales ranged from 0.74 for self-efficacy after the visit to 0.94 for perceived benefits after the visit.

We measured current stage of readiness for FOBT and colonoscopy by asking whether patients were currently (1) not considering completing the test (precontemplative); (2) thinking about completing the test (contemplative); or (3) planning to 
do the test (planning). We inquired about FOBT and colonoscopy separately, which yielded separate stages of readiness for each test. We did not assess stage of readiness with regard to flexible sigmoidoscopy because staff physicians do not often recommend it.

We used handheld digital recorders to audiorecord all encounters, which assistants placed in unobtrusive but unconcealed locations in the examination room. Two research assistants independently listened to audio-recordings to identify discussions of CRC screening. An assistant then transcribed CRC screening discussions.

\section{Coding of Conversation Content}

We developed a codebook to guide the recognition of counseling relevant to behavioral constructs. We included examples of provider statements addressing specific constructs that we believed would be common or typical (eg, discussion of the benefit of early detection and removal of polyps). Regarding self-efficacy, coders assessed whether providers made statements that research and theory suggest may contribute to enhanced patient confidence when undergoing CRC screening. These included direct inquiries regarding confidence; expressions of confidence in the patient; referring to patients' prior successes with other health behaviors; attempts to reframe prior failures to complete screening as learning experiences; and explicit explanations of the next steps the patient must take to complete screening. ${ }^{18} \mathrm{We}$ iteratively revised the codebook based on pilot coding of small sets of transcripts.

An investigator (JJF) and an assistant independently coded all transcripts for the presence of counseling that addressed constructs and determined which types of CRC tests were discussed. Coders were blinded to provider identity and to before-visit patient data. Thus, they could not code whether counseling related to patient attitudes and beliefs was tailored to respond to explicit or implicit patient concerns or requests. Interrater agreement (Cohen's $\kappa$ ) regarding the presence or absence of construct-specific content ranged from 0.44 for benefits to 0.57 for perceived barriers. Disagreements between coders were resolved by discussion and consensus.

We categorized encounters based on (1) whether or not CRC screening was discussed; (2) whether or not providers addressed one of the following constructs: benefits, barriers, risk/susceptibility, or self-efficacy; and (3) whether CRC screening discussions addressed one or more of these constructs.

\section{Outcomes}

We considered patients to have advanced in stage of readiness if they moved from a precontemplative or contemplative stage to a more advanced stage for either colonoscopy or FOBT. Six months after the visit we performed a standardized review of the electronic chart to ascertain whether CRC screening tests were ordered during visits and whether patients had completed the ordered tests.

\section{Patient Covariates}

Questionnaires before the visit included questions regarding race/ethnicity, education, annual household income, health status (five-point Likert scale from "poor" to "excellent"), and current smoking. We abstracted age and sex from the electronic record.

\section{Analyses}

We performed descriptive analyses to characterize patients and CRC screening conversations with providers. We used Fisher's exact tests to assess bivariate associations between CRC screening discussions and advancement in stage of readiness, test ordering, and completion. We used analysis of covariance to assess whether CRC screening discussions were associated with adjusted mean differences in after-visit domain scores for perceived benefits, barriers, susceptibility, self-efficacy, and intention. Because scales before and after the visit were constructed by averaging across individual standardized items (with means of 0 and standard deviations of 1 ), parameter estimates ( $\beta$ coefficients) from analysis of covariance models estimate of mean differences in after-visit scale scores between patients with and without CRC screening discussions while adjusting for corresponding scale scores before the visit and other patient covariates. Confidence intervals and hypothesis tests were based on robust standard errors to account for clustering of patients within providers.

We used exact logistic regression to assess whether CRC screening discussions were associated with binary outcomes (advancement in stage of readiness and CRC test completion), adjusting for covariates. Exact methods provide more accurate 
inferences than conventional unconditional maximum likelihood methods for binary outcomes with very low or zero cell sizes. We restricted analyses for advancement in stage of readiness to patients who did not report before visits that they planned to undergo FOBT and colonoscopy.

In all regression models, we adjusted for health status and household income $(<\$ 50,000$ per year vs $\geq \$ 50,000$ per year), selecting these covariates based on a priori theory-based considerations. In the logistic regression models, we also adjusted for stages of readiness before the visit to undergo FOBT and colonoscopy. In bivariate analyses (not shown), no other patient characteristics were associated with CRC screening discussion. We found no meaningful difference in outcome based on whether the patient or the provider initiated the CRC screening discussion. Hence, to avoid bias from overfitting in the setting of small samples, ${ }^{19}$ no further covariates were included. We conducted analyses using STATA SE (version 11.0, StataCorp, College Station, TX), and hypothesis tests were two-sided, with $\alpha=0.05$.

\section{Results}

\section{Providers and Patients}

During the study period, 57 patients provided written informed consent to participate, and 50 patients within 20 providers were eligible with complete analytic data (Figure 1). The median number of patients per provider was three (range, one to five patients). Participating patients were predominantly women, and most had attended college (Table 1).

\section{CRC Screening Conversations}

Patients and providers discussed CRC screening in 38 of the 50 visits ( $76 \%$; Table 2). The median total time spent discussing CRC screening was 2.6 minutes. Physicians described one or more test options during most discussions. When specific tests were discussed, colonoscopy was always mentioned as an option, whereas flexible sigmoidoscopy and FOBT were mentioned in fewer than half of the conversations. Provider counseling addressed one or more behavioral constructs in more than two thirds of conversations and addressed two or more constructs in nearly half (Table 2). When addressing constructs, providers discussed the benefits of CRC screening in more than half of conversations; less commonly they addressed risk/susceptibility, barriers, or self-efficacy. The frequency and content of CRC screening discussions were similar among faculty versus residents, physicians versus physician assistant/family nurse practitioners, and family physicians versus internists (data not shown).

\section{Figure 1. Flow of study patients.}

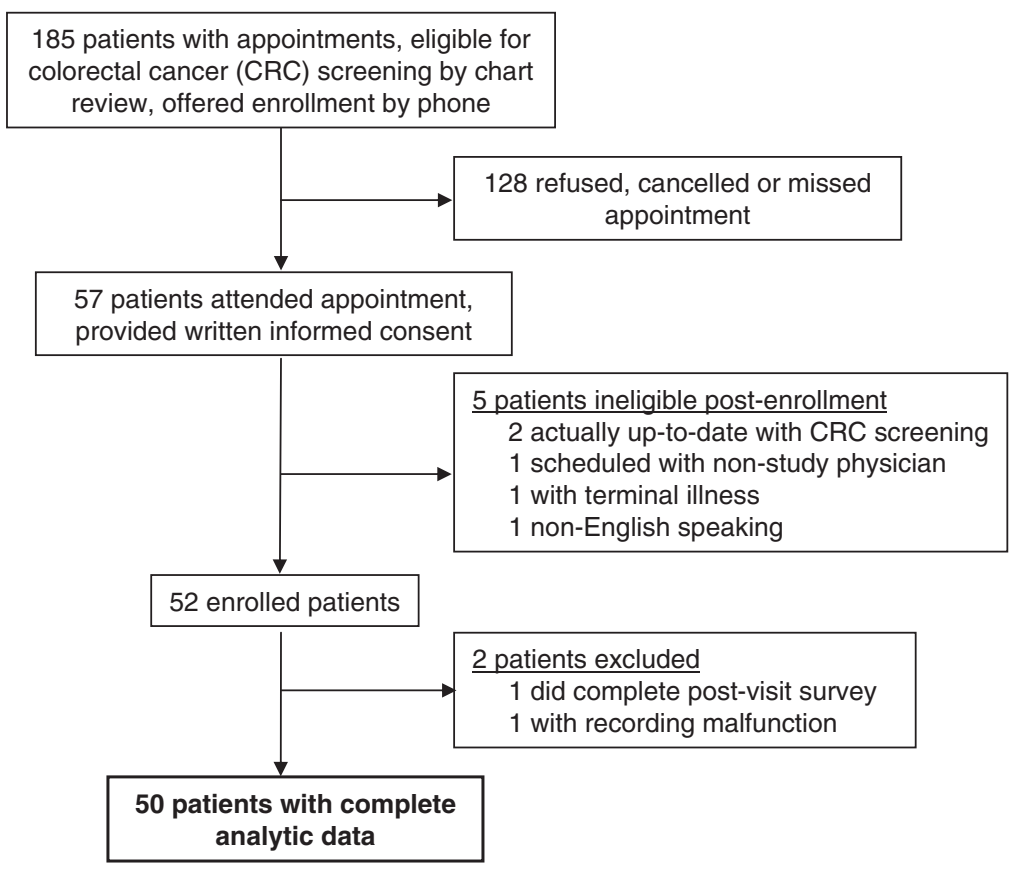


Table 1. Patient Characteristics $(n=50)$

\begin{tabular}{lc}
\hline Characteristic & \\
Age, years (mean [SD]) & $57.3(6.2)$ \\
Female & $66(33)$ \\
Race/ethnicity & \\
Non-Hispanic white & $29(58)$ \\
African American & $13(26)$ \\
Hispanic & $5(10)$ \\
Other & $3(6)$ \\
Education & \\
High school, GED, or less & $8(16)$ \\
Some college (no degree) & $24(48)$ \\
Graduated from college & $18(36)$ \\
Household income (\$) & \\
$<$ 20,000 & $12(24)$ \\
20,00-49,999 & $7(14)$ \\
$50,000-84,999$ & $16(32)$ \\
$\geq 85,000$ & $12(24)$ \\
Missing & $3(6)$ \\
Self-reported health status & \\
Poor & $1(2)$ \\
Fair & $14(28)$ \\
Good & $20(39)$ \\
Very good & $11(22)$ \\
Excellent & $4(8)$ \\
Burrent smoker & $18(36)$ \\
$\quad$ Precoline stage of readiness with regard & \\
Contemplation & \\
Planning & $10(20)$ \\
\hline & $28(56)$ \\
& $12(24)$ \\
\hline
\end{tabular}

Values provided as n (\%) unless otherwise indicated. GED, general educational development.

Providers typically addressed constructs with short, unelaborated statements. For example, one provider addressed screening benefits by saying, "You can treat colon cancer in its early stage and prevent it from becoming a kind of cancer that would need a more extensive surgery." Another provider addressed barriers by saying, "Statistically, depending on the operator, the chance of there being a perforation ... with the sigmoidoscopy is about one in 6000 to 8000 . For [colonoscopy], it's about one in 4000 to 6000 . So a very, very small percentage. ..." In contrast, conversations that did not address constructs often consisted of a brief test description, punctuated by the provider's offer to order testing.

\section{Patient Attitudes and Intention}

CRC screening discussions were not associated with significant changes in perceived benefits, bar- riers, or self-efficacy after the visit, even when conversations included content related to these constructs (Table 3). However, CRC screening discussions were significantly associated with increased perceived risk/susceptibility to CRC ( $\beta=$ $0.39 ; 95 \%$ CI, $0.09-0.68)$. This increase was of similar magnitude whether or not counseling addressed patient risk/susceptibility. Similarly, discussion of CRC screening was significantly associated with increased patient intention to undergo screening ( $\beta=0.42 ; 95 \% \mathrm{CI}, 0.11-0.73$ ), with statistically similar increases between discussions that $\operatorname{did}(\beta=0.44 ; 95 \%$ CI, $0.12-0.75)$ and did not $(\beta=0.41 ; 95 \%$ CI, 0.05-0.77) address behavioral constructs. The observed increases in perceived risk/susceptibility and intention correspond to medium to large effect sizes. ${ }^{20}$

\section{Test Planning, Ordering, and Completion}

Among patients who were not already planning to undergo both FOBT and colonoscopy before visits, discussion of CRC screening was significantly associated with advancement in stage of readiness

Table 2. Characteristics of Conversations $(n=38)$ Regarding Colorectal Cancer Screening

\begin{tabular}{lc}
\hline Characteristic & \\
Who initiated CRC screening conversation & $27(71)$ \\
Provider & $11(29)$ \\
Patient & \\
Discussion of specific tests & $2(5)$ \\
$\quad$ General discussion but no specific test mentioned & $36(95)$ \\
Specific tests discussed & $36(95)$ \\
$\quad$ Colonoscopy & $15(39)$ \\
$\quad$ Flexible sigmoidoscopy & $12(32)$ \\
Fecal occult blood testing & $2(5)$ \\
$\quad$ CT colonography & \\
Discussion of behavioral constructs & $20(53)$ \\
Benefits of screening & $11(29)$ \\
Common patient-level barriers & $14(37)$ \\
Patient's risk and susceptibility to CRC & \\
Patient self-efficacy to complete screening & 0 \\
$\quad$ Provider explored patient's confidence & 0 \\
Provider referred to prior successful behaviors & 0 \\
Provider reframed prior failures & $7(18)$ \\
Provider specified next steps & 0 \\
Providers expressed confidence in patient & $26(68)$ \\
One or more behavioral constructs & $17(45)$ \\
Two or more behavioral constructs & \\
\hline
\end{tabular}

Values provided as $\mathrm{n}(\%)$ unless otherwise indicated. CRC, colorectal cancer; CT, computed tomography. 
Perceived benefits (SD, 0.86)

No discussion of CRC screening

Any discussion

No discussion of CRC screening

$$
-0.02(-0.35 \text { to } 0.31)
$$

$$
\text { Ref }
$$

Screening discussed but not benefits

Benefits discussed

-0.04 ( -0.40 to 0.32$)$

Perceived barriers ${ }^{\dagger}$ (SD, 0.63)

No discussion of CRC screening

Any discussion

$0.00(-0.39$ to 0.38$)$

No discussion of CRC screening

$$
\begin{gathered}
\text { Ref } \\
0.00(-0.15 \text { to } 0.15)
\end{gathered}
$$

$$
\text { Ref }
$$

Screening discussed but not barriers

$0.04(-0.12$ to 0.21$)$

Barriers discussed

$-0.11(-0.32$ to 0.10$)$

Perceived risk/susceptibility (SD, 0.94)

No discussion of CRC screening

Ref

Any discussion

$0.39(0.09-0.68)$

No discussion of CRC screening

Ref

Screening discussed but not risk/susceptibility

$0.37(0.04-0.70)$

Risk/susceptibility discussed

$0.42(0.13-0.72)$

Self-efficacy to undergo CRC screening (SD, 0.70)

No discussion of CRC screening

Any discussion

No discussion of CRC screening

Screening discussed but not next steps

Next steps specified

Intention to undergo CRC screening (SD, 0.78)

No discussion of CRC screening

Any discussion

No discussion of CRC screening

Screening discussed but no constructs

$$
\text { Ref }
$$$$
-0.06(-0.42 \text { to } 0.29)
$$

Ref

$-0.06(-0.42$ to 0.30$)$

$-0.08(-0.52$ to 0.37$)$

One or more constructs addressed

Ref

$0.42(0.11-0.73)$

Ref

$0.44(0.12-0.75)$

$0.41(0.05-0.77)$

* $\beta$ coefficients estimate adjusted mean differences (vs reference category) in construct-specific after-visit scale scores using analysis of covariance models to adjust for measures of the relevant behavioral construct, health status (fair/poor vs. good/very good/excellent), and annual household income $(<\$ 50,000$ vs. $\geq \$ 50,000)$ before the visit. Confidence intervals are corrected for within-physician clustering by use of robust standard errors.

${ }^{\dagger}$ Higher numbers on the barriers scale indicate greater perceived barriers, so negative coefficients imply lower perceived barriers after versus before the visit.

CRC, colorectal cancer.

(Table 4). Providers never ordered CRC screening during visits when CRC screening was not discussed, but they did order screening tests after most CRC screening discussions. Among 31 patients who had tests ordered, $26(84 \%)$ were referred for colonoscopy, whereas five (16\%) were referred for FOBT without other tests. The proportions of patients who advanced in stage of readiness or had tests ordered were similar whether or not counseling addressed behavioral constructs. At the 6-month follow-up, none of the 13 patients who did not receive counseling related to CRC screening during study visits completed screening compared with 8 of 12 patients (67\%) who discussed screening but not constructs and 9 of 26 patients $(35 \%)$ who discussed constructs (Fisher's exact test for overall difference; $P=.001)$. All completed $\mathrm{CRC}$ tests were ordered during study visits; none were ordered during visits later during the 6-month follow-up. 


\begin{tabular}{|c|c|c|c|c|c|}
\hline \multirow[b]{2}{*}{ Outcome } & \multirow[b]{2}{*}{ Overall } & \multicolumn{3}{|c|}{ CRC Screening Discussion } & \multirow[b]{2}{*}{$P$} \\
\hline & & $\begin{array}{c}\text { No } \\
\text { Discussion }\end{array}$ & $\begin{array}{l}\text { Screening Discussed } \\
\text { Without Addressing } \\
\text { Behavioral Constructs }\end{array}$ & $\begin{array}{l}\text { One or More } \\
\text { Constructs } \\
\text { Addressed }\end{array}$ & \\
\hline $\begin{array}{l}\text { Advancement in stage of readiness to undergo } \\
\text { CRC screening }(\mathrm{N}=40)^{*}\end{array}$ & $22 / 40(55)$ & $1 / 8(13)$ & $8 / 11(73)$ & $13 / 21(62)$ & .03 \\
\hline CRC screening ordered $(\mathrm{N}=50)$ & $31 / 50(62)$ & $0 / 12(0)$ & $9 / 12(75)$ & $22 / 26(85)$ & $<.001$ \\
\hline CRC completed $(\mathrm{N}=50)$ & $17 / 50(33)$ & $0 / 12(0)$ & $8 / 12(67)$ & $9 / 26(35)$ & .001 \\
\hline
\end{tabular}

Values provide as $\mathrm{n} / \mathrm{N}(\%)$.

*Defined as more advanced stage of readiness with regard to a CRC screening test after the visit (compared with before the visit); the analysis for this outcome was restricted to the 40 patients who did not report on questionnaires before the visit that they were planning to undergo fecal occult blood testing and colonoscopy.

CRC, colorectal cancer.

In exact logistic regression analyses, CRC screening discussions remained significantly associated with advancement in stage of readiness and completion of CRC screening after adjusting for patient covariates (Table 5). The association between discussions and advancement of stage of readiness was similar whether or not providers addressed constructs. Contrary to our hypothesis, discussions that did not address constructs were more strongly associated with CRC completion than conversations that did address constructs, although confidence intervals were wide and overlapping.

\section{Discussion}

Among this sample of patients eligible for CRC screening, a conversation with a primary care provider regarding CRC screening was strongly associated with increased perceived susceptibility to CRC, advancement in intention and stage of readiness to undergo screening, and ultimate completion of screening. Although most discussions included content that we hypothesized might facilitate CRC screening uptake based on behavioral theory, CRC screening discussions were associated with favorable outcomes whether or not discussions included such content. The strong association between conversations that did not address behavioral constructs and CRC screening completion suggests that provider counseling regarding CRC screening may not need to be highly nuanced to effectively promote screening. As has been shown for smoking cessation and reduction of alcohol intake, ${ }^{21,22}$ brief, direct encouragement from providers may have a clinically important impact on patients' CRC screening behavior.
At the same time, there are other plausible explanations for why CRC screening discussions were associated with similar outcomes, whether or not they addressed constructs. First, study providers

Table 5. Adjusted Association between Provider Conversation and Advancement in Stage of Readiness and Colorectal Cancer Screening Completion

\begin{tabular}{lc}
\hline Independent Variable & OR $(95 \% \mathrm{CI})^{\dagger}$ \\
\hline $\begin{array}{c}\text { Advancement in stage of } \\
\text { readiness }\left(\mathrm{n}=40^{*}\right)\end{array}$ & \\
No discussion of CRC screening & $1.0(\mathrm{ref})$ \\
Any discussion of CRC screening & $12.7(1.3-682.0)$ \\
No discussion of CRC screening & $1.0(\mathrm{ref})$ \\
Screening discussed without & $14.5(1.04-986.6)$ \\
$\quad$ addressing behavioral constructs & \\
One or more constructs addressed & $11.0(1.06-602.2)$ \\
CRC screening completed (n $=50)$ & \\
No discussion of CRC screening & $1.0(\mathrm{ref})$ \\
Any discussion of CRC screening & $10.0(1.4-\infty)$ \\
No discussion of CRC screening & $1.0(\mathrm{ref})$ \\
Screening discussed without & $33.2(3.6-\infty)$ \\
$\quad$ addressing behavioral constructs & \\
One or more constructs addressed & $5.8(0.7-\infty)$ \\
\hline
\end{tabular}

*Analyses for this outcome was restricted to the 40 patients who did not report on questionnaires before the visit that they were planning to undergo fecal occult blood testing and colonoscopy during. Advancement in stage of readiness is defined as more advanced stage of readiness with regard to one of these tests post-visit as compared to pre-visit.

${ }^{\dagger}$ Odds ratios were estimated using exact logistic regression and adjusted for health status (fair/poor vs good/very good/excellent), annual household income $(<\$ 50,000$ vs $\geq \$ 50,000)$, and stages of readiness before the visit with regard to fecal occult blood testing and colonoscopy. The upper $95 \% \mathrm{CI}$ may be unbounded in the setting of zero cells (i.e., when no patients completed CRC screening in the absence of CRC screening discussions).

CRC, colorectal cancer. 
may not have tailored discussion of constructs to address patient concerns. Counseling tailored to patients' foremost concerns may conceivably be more effective than nontailored discussion relating to behavioral constructs. Second, it is possible that usual provider counseling is ineffectual in addressing constructs because of inordinate haste, lack of skill, or both. Third, patients may perceive more nuanced counseling as ambiguous or tentative compared with direct, simple recommendations. A recent survey suggested that physicians discussion of multiple test options for CRC screening may confuse patients and steer some away from screening. ${ }^{23}$ Similarly, within a Veterans Affairs primary care clinic, there was an inverse association between the use of informed decision making during counseling and CRC screening completion. ${ }^{24} \mathrm{~A}$ randomized study comparing direct, unequivocal physician recommendations with more nuanced, theory-driven alternatives would elucidate how physicians can best promote CRC screening during office visits.

Our findings add to a limited literature regarding the content of physician counseling related to CRC screening. A retrospective survey of Midwest health plan enrollees suggested that discussions may be more effective if physicians provide appointments for CRC screening or arrange follow-up to give patients screening results, ${ }^{25}$ neither of which commonly occurred during study visits. An analysis of 18 videotaped discussions of CRC screening suggested that physician counseling regarding CRC screening is typically brief and descriptive, usually omitting any discussion of benefits and risks or elicitation of patients' views. ${ }^{26}$ Though our study suggests that primary care providers often discuss constructs related to CRC screening, discussions were often limited in scope. As in other US-based studies, ${ }^{27-30}$ study providers demonstrated a clear preference for screening colonoscopy over flexible sigmoidoscopy or FOBT, which were discussed in a minority of visits.

CRC screening discussions were associated with a medium to large increase in patients' perceived risk/susceptibility to CRC, even when they did not specifically address risk or susceptibility. It is possible that the mere discussion of CRC screening in the context of an office visit is sufficient to increase many patients' perceptions of CRC risk. Meanwhile, we found no evidence that domain-specific counseling increased perceived benefits or self-efficacy or reduced perceived barriers to CRC screening, although providers addressed self-efficacy to a limited extent. Providers also tended to emphasize the benefits of screening, whereas other constructs, such as barriers, may conceivably motivate screening more powerfully.

Study findings must be interpreted in light of limitations. First, the agreement between coders regarding the presence of counseling related to constructs was moderate, and measurement error may have obscured differences in outcomes after counseling that did and did not address constructs. Second, the small cohort size yielded low statistical precision in some analyses, and readers should consider not only point estimates but confidence intervals. Third, relatively few conversations addressed multiple constructs, limiting our ability to compare more versus less highly nuanced conversations. Fourth, providers may have been more likely to address constructs during visits with more resistant patients or to discuss CRC screening with patients who were more likely to undergo screening. Although analyses adjusted for baseline intention or stage of readiness, confounding by indication of this sort may affect results. Fifth, CRC screening discussions may have been prompted or modified by study conditions, including unblinded audio recordings. Also, we lacked information about nonverbal or affective dimensions of provider communication. Finally, our study included academically affiliated primary care practices at a single institution, and results may not generalize to other practices.

\section{Conclusion}

Primary care providers in this study typically provided relatively brief counseling regarding CRC screening that emphasized colonoscopy. Provider counseling was strongly associated with increased perceived risk and susceptibility to CRC, advancement in patient intention, and ultimate completion of CRC screening whether or not it addressed behavioral constructs. These findings suggest that brief physician recommendations may motivate patients to complete CRC screening even without addressing behavioral constructs. Ideally, these findings would be confirmed within a randomized trial conducted in diverse primary care practices.

\footnotetext{
The authors acknowledge the assistance of staff at the University of California, Davis, Center for Health Care Policy and Research, including Colleen Cameron, Teresa Farley, and Laura Sterner.
} 


\section{References}

1. Smith RA, Cokkinides V, Brawley OW. Cancer screening in the United States, 2008: a review of current American Cancer Society guidelines and cancer screening issues. CA Cancer J Clin 2008; 58(3):161-79.

2. U.S. Preventive Services Task Force. Screening for colorectal cancer. 2008. Available at: http://www. ahrq.gov/clinic/uspstf08/colocancer/colors.htm. Accessed 1 June 2009.

3. Winawer S, Fletcher R, Rex D, et al. Colorectal cancer screening and surveillance: clinical guidelines and rationale-Update based on new evidence. Gastroenterology 2003;124(2):544-60.

4. Centers for Disease Control and Prevention. Use of colorectal cancer tests-United States, 2002, 2004, and 2006. MMWR Morb Mortal Wkly Rep 2008; 57(10):253-8.

5. Brenes GA, Paskett ED. Predictors of stage of adoption for colorectal cancer screening. Prev Med 2000; 31(4):410-6.

6. Klabunde CN, Schenck AP, Davis WW. Barriers to colorectal cancer screening among Medicare consumers. Am J Prev Med 2006;30(4):313-9.

7. Zapka JG, Puleo E, Vickers-Lahti M, Luckmann R. Healthcare system factors and colorectal cancer screening. Am J Prev Med 2002;23(1):28-35.

8. Myers RE, Trock BJ, Lerman C, Wolf T, Ross E, Engstrom PF. Adherence to colorectal cancer screening in an HMO population. Prev Med 1990; 19(5):502-14.

9. Vernon SW, Myers RE, Tilley BC. Development and validation of an instrument to measure factors related to colorectal cancer screening adherence. Cancer Epidemiol Biomarkers Prev 1997;6(10):825-32.

10. Rawl S, Champion VL, Menon U, Loehrer PJ, Vance GH, Skinner CS. Validation of scales to measure benefits of and barriers to colorectal cancer screening. J Psychosoc Oncol 2001;19:47-63.

11. Rawl SM, Menon U, Champion VL, et al. Do benefits and barriers differ by stage of adoption for colorectal cancer screening? Health Educ Res 2005;20(2):137-48.

12. Janz NK, Lakhani I, Vijan S, Hawley ST, Chung LK, Katz SJ. Determinants of colorectal cancer screening use, attempts, and non-use. Prev Med 2007;44(5):452-8.

13. Bazargan M, Ani C, Bazargan-Hejazi S, Baker RS, Bastani R. Colorectal cancer screening among underserved minority population: discrepancy between physicians' recommended, scheduled, and completed tests. Patient Educ Couns 2009;76(2):240-7.

14. Pignone M, Harris R, Kinsinger L. Videotape-based decision aid for colon cancer screening. A randomized, controlled trial. Ann Intern Med 2000;133(10): 761-9.

15. Jerant A, Kravitz RL, Rooney M, Amerson S, Kreuter M, Franks P. Effects of a tailored interactive multimedia computer program on determinants of colorectal cancer screening: a randomized controlled pilot study in physician offices. Patient Educ Couns 2007;66(1):67-74.

16. Prochaska JO. Systems of Psychotherapy: A Transtheoretical Analysis. Pacific Grove, CA: Brooks-Cole; 1979.

17. Prochaska JO, Velicer WF, Rossi JS, et al. Stages of change and decisional balance for 12 problem behaviors. Health Psychol 1994;13(1):39-46.

18. Jerant A, Kravitz RL, Azari R, et al. Training residents to employ self-efficacy-enhancing interviewing techniques: randomized controlled trial of a standardized patient intervention. J Gen Intern Med 2009;24(5):606-13.

19. Peduzzi P, Concato J, Kemper E, Holford TR, Feinstein AR. A simulation study of the number of events per variable in logistic regression analysis. J Clin Epidemiol 1996;49(12):1373-9.

20. Cohen J. Statistical Power Analysis for the Behavioral Sciences. 2nd edition. Hillsdale, NJ: Lawrence Erlbaum Associates; 1988.

21. Kaner EF, Beyer F, Dickinson HO, et al. Effectiveness of brief alcohol interventions in primary care populations. Cochrane Database Syst Rev 2007(2): CD004148.

22. Stead LF, Bergson G, Lancaster T. Physician advice for smoking cessation. Cochrane Database Syst Rev 2008(2):CD000165.

23. Jones RM, Vernon SW, Woolf SH. Is discussion of colorectal cancer screening options associated with heightened patient confusion? Cancer Epidemiol Biomarkers Prev 2010;19(11):2821-5.

24. Ling BS, Trauth JM, Fine MJ, et al. Informed decision-making and colorectal cancer screening: is it occurring in primary care? Med Care 2008;46(9 Suppl 1):S23-9.

25. Lafata JE, Divine G, Moon C, Williams LK. Patientphysician colorectal cancer screening discussions and screening use. Am J Prev Med 2006;31(3):202-9.

26. Wolf MS, Baker DW, Makoul G. Physician-patient communication about colorectal cancer screening. J Gen Intern Med 2007;22(11):1493-9.

27. Klabunde CN, Lanier D, Nadel MR, McLeod C, Yuan G, Vernon SW. Colorectal cancer screening by primary care physicians: recommendations and practices, 2006-2007. Am J Prev Med 2009;37(1):8-16.

28. McQueen A, Bartholomew LK, Greisinger AJ, et al. Behind closed doors: physician-patient discussions about colorectal cancer screening. J Gen Intern Med 2009;24(11):1228-35.

29. Phillips KA, Liang SY, Ladabaum U, et al. Trends in colonoscopy for colorectal cancer screening. Med Care 2007;45(2):160-7.

30. Fenton JJ, Cai Y, Green P, Beckett LA, Franks P, Baldwin LM. Trends in colorectal cancer testing among Medicare subpopulations. Am J Prev Med 2008;35:194-202. 